INVESTIGACIÓN

Recibido: 16/03/2021 --- Aceptado: 16/03/2021 --- Publicado: 24/05/2021

\title{
PERCEPCIÓN DE LOS ESTUDIANTES DEL GRADO DE PUBLICIDAD Y RR.PP. SOBRE LA RELACIÓN ENTRE MARCAS E INFLUENCERS EN INSTAGRAM
}

\section{Advertising students' perception about the relationship between brands and influencers on Instagram}

Alberto Martín García. Universidad de Valladolid. España. alberto.martin.garcia@uva.es

Referencia de financiación. Proyecto "Verdad y ética en las redes sociales (INTERNÉTICA)". Convocatoria de proyectos $I+D+I$ del Ministerio de Ciencia, Innovación y Universidades de 2019 (PID2019-104689RB-100)

Cómo citar el artículo:

Martín García, A. (2021). Percepción de los estudiantes del grado de publicidad y RR.PP. Sobre la relación entre marcas e influencers en Instagram. Vivat Academia. Revista de Comunicación, 154, 57-78. http:/ / doi.org/10.15178/va.2021.154.e1337 http://www.vivatacademia.net/index.php/vivat/article/view/1337

\section{RESUMEN}

La irrupción de los influencers en las redes sociales como generadores de contenido ha supuesto un cambio en la estrategia publicitaria de las marcas en el entorno digital, siendo ellos los que desempeñan la función de emisores del contenido comunicativo a reproducir de forma persuasiva. Este artículo trata de explorar la percepción que los usuarios de redes sociales con edades comprendidas entre 18-29 años, y que estudian o han estudiado el Grado en Publicidad y RR.PP., tienen de la presencia de los influencers en Instagram, y si el impacto que éstos generan en la relación entre el potencial consumidor y la marca es positivo o se vuelve contraproducente para la entidad. Para ello se ha aplicado una metodología cuantitativa consistente en un cuestionario completado por doscientos treinta jóvenes de ambos sexos y residentes en España. Los resultados revelan una tendencia mayoritaria en la población cuestionada de percibir que la relevancia que ejercen sobre ella los influencers se traduce parcialmente en una mejora de la relación con la marca y en un incremento del deseo de consumo del producto o servicio mostrado en Instagram. Este estudio representa una aportación analítica sobre cómo el alumnado de una titulación relacionada con el contenido del estudio percibe una realidad comunicativa a la que 
están habituados a tratar en el día a día, y supone un punto de partida para posteriores ampliaciones que impliquen a otros sectores que no estén directamente relacionados con la comunicación.

PALABRAS CLAVE: Redes sociales - neuromarketing - influencers - Instagram comunicación - publicidad - marcas - estrategia - universidad.

\section{ABSTRACT}

The appearance of influencers to social media as content generators has meant a change in the advertising strategy of brands in the digital environment, being they who play the role of transmitters of the media content to be reproduced persuasively. This article tries to explore the perception that users of social media aged between 1829 years, who study or have studied a Degree in Advertising and Public Relations, have of the presence of influencers on Instagram, and if the impact that they generate in the relationship between the potential consumer and the brand is positive or becomes negative for the entity. For this, a quantitative methodology has been applied consisting of a questionnaire completed by two hundred and thirty young people of both sexes and residents in Spain. The results reveal a main tendency in the questioned population to perceive that the relevance of influencers exert on them translate into an improvement in the relationship with the brand and an increase in the desire to consume the product or service shown on Instagram. This study represents an analytical contribution on how the students of a degree related to the content of the study perceive a media reality that they are used to deal with on a day-to-day basis, and it is a starting point for subsequent extensions that involve other sectors that are not directly related to communication.

KEYWORDS: Social media - neuromarketing - influencers - Instagram communication - advertising - brands - strategy - university.

\section{INTRODUCCIÓN}

La consolidación de Instagram, marca perteneciente a Facebook, como la red social más popular entre la gente joven en España y la más idónea para seguir a los influencers (IAB, 2020), ha creado una nueva forma de relación entre el público objetivo y la marca, necesitando ésta la labor de dichos influencers como mediadores para hacer llegar el mensaje de la compañía a su potencial consumidor. Se denomina influencer a una "persona que cuenta con cierta credibilidad sobre un tema concreto, y por su presencia e influencia en redes sociales puede llegar a convertirse en un prescriptor interesante para una marca" (García, 2018). Las marcas no son ajenas al cambio tecnológico y a la evolución en la forma de llegar a sus futuros consumidores. No gozan de espacios reservados para su comunicación, sino que "están obligadas a participar en los medios tal como lo hacen los usuarios comunes, ya que la interacción a través de redes sociales es un distintivo de esta forma de publicidad." (Hernández-Fernández, 2013, p. 58). Dentro del concepto se establecen subcategorías en función del número de fans que tiene cada persona en sus redes sociales: nanoinfluencers (de 1.000 a 10.000 seguidores), 
microinfluencers (de 10.000 a 100.000 seguidores), macroinfluencers (de 100.000 a un millón de seguidores) y megainfluencers, con más de un millón de seguidores (García, 2020). Si bien tomamos esta clasificación como acertada, no existe una tabla oficial por cantidad de seguidores, por lo que en diferentes análisis pueden encontrarse clasificaciones que difieran del que citamos.

Instagram se posiciona en los últimos años como la red social en la que las marcas consiguen una mayor cuota de interacciones (67\%), de engagement $(78 \%$ ) y de eficiencia $(72 \%)(\mathrm{IAB}, 2020)$. De esta manera, los datos más cuantitativos referentes a la cantidad de usuarios que interactúan en esta red social se ven apoyados por otros más cualitativos que hacen referencia a la participación y a la eficacia del mensaje transmitido. Sin embargo, hay datos como el engagement, que hace alusión al grado de interés en forma de participación que consigue una publicación en una red social, que tienen que ser tratados con cierta relatividad: no encontramos ni a nivel profesional ni académico una fórmula unívoca y ampliamente utilizada. "Cada empresa mide el compromiso de su audiencia cuantificando las interacciones de la forma en que considera conveniente" (Navío-Navarro, 2021, p. 242).

Para valorar ese compromiso ligado no se recurre únicamente al terreno cuantitativo de los datos "sino más a la calidad de sus aportaciones, de sus interacciones, de la reputación acreditada" (Morales, 2019, p. 75). "Los factores de personalidad que más explican quién usa y cómo se usa Instagram son la extraversión y la apertura a nuevas experiencias" (Casado y Carbonell, 2018, p. 29), y partiendo de ese conocimiento se crean las estrategias comunicativas y publicitarias para que las marcas recurran a los influencers y se alejen en las redes sociales de la publicidad más tradicional.

"No es el propio usuario quien se convierte en influencer, sino que son los demás los que nos hacen más o menos influyentes" (Morales, 2019, p. 75). No son un resultado de la presencia de las marcas en Instagram u otra red social, sino que la popularidad adquirida los ha convertido en un soporte para dar a conocer y promocionar sus productos y servicios. Es decir, "actúan como marcas propias y desarrollan su propia identidad, muestran rasgos de su personalidad, utilizan un tono para dirigirse a su comunidad y se asocian a unos valores, imagen y colores corporativos." (FernándezLerma, 2017, p. 19)

Para lograr esa afinidad entre marca y usuario a través de los influencers, primero la marca tiene que conocer los motivos que llevan a las personas a usar una red social como Instagram. Conocerlos es tan importante como hacerlo sobre el funcionamiento de la propia herramienta y las posibilidades que ofrece. Hay cuatro razones principales por los que se utiliza Instagram actualmente: a) vigilancia/control y conocimiento de la vida de los demás; b) a modo de documentación (relacionada con el uso de recopilar recuerdos); c) ser popular; d) creatividad (Sheldon y Bryant, 2016). Sobre este último aspecto trabajan especialmente las marcas para sorprender continuamente a los seguidores en un entorno digital en el que hay gran cantidad de opciones y de

Vivat Academia. Revista de Comunicación. 2021, nº 154, 57-78 
competencia. Aunque se intuye en las categorías que nos proponen Sheldon y Bryant, conviene ahondar en la importancia de las relaciones interpersonales como uno de los elementos que motiva el uso de Instagram, tanto con gente del entorno como con desconocidos. Y desde la vertiente más negativa, reseñamos que Instagram "presenta ciertos componentes adictivos como la inmediatez por ser el primer usuario en comentar los "likes" en las publicaciones, visualizaciones de vídeos en directo, conseguir mayor número de seguidores" (Romero et al., 2019, p. 91). Estos elementos adictivos llevan a un crecimiento año tras año del consumo de esta red social (IAB, 2020).

Al igual que los motivos de consumo de Instagram, antes de recurrir a un influencer en concreto las marcas tienen que conocer los formatos comunicativos que ofrece la aplicación. Estos son 1) Anuncios en stories. 2) Anuncios con foto. 3) Anuncios con vídeo. 4) Anuncios por secuencia (Business Instagram, s.f.). La elección de uno u otro dependerá del contenido a comunicar y del tiempo que la marca necesita para lograr que su mensaje cale. Su acierto marcará que los indicadores relacionados con el engagement o la eficacia sean mayores.

No vamos a profundizar en qué es el marketing, pero sí conviene recordar de cara a este estudio que es el proceso por el cual las empresas crean un valor material o inmaterial para los clientes y construyen relaciones sólidas con los propios clientes con el fin de lograr como contraprestación el valor de dichos clientes (Kotler y Armstrong, 2014). Para crear esas relaciones, es necesario establecer un plan de comunicación para hacer una campaña con influencers que impacte en el público. Se pone de manifiesto que en las redes sociales al igual que en formatos más tradicionales, como puede ser una campaña publicitaria en televisión, las marcas han de recurrir a una estrategia previamente marcada: “1) Establecer objetivos. 2) Buscar el perfil idóneo. 3) Ser coherente con la marca. 4) Respetar la personalidad del influencer. 5) Decidir si la acción es individual o coral. 6) Construir un mensaje claro. 7) Plantear acuerdos win-win. 8) Comunicación $360^{\circ}$. 9) Evaluación y reajuste constante. 10) Medir el retorno." (Universidad Oberta de Catalunya, 2018). La rapidez del mundo digital hace imperativo estos pasos para la marca de cara a controlar al máximo la acción comunicativa y ser eficaz. Hay que recordar que esa comunicación $360^{\circ}$ consiste en utilizar diferentes canales y recursos publicitarios, las acciones con influencers en Instagram son únicamente una parte más del conjunto de piezas audiovisuales a publicar por la marca. "Un parámetro de vital importancia a considerar al analizar la figura del prescriptor publicitario es apostar por una verdadera y creíble relación en primera instancia entre el producto y el propio famoso" (Castelló y Del Pino, 2019, p. 31), relación en la que ambos juegan un papel fundamental desde el punto de vista publicitario.

La renovación de contenidos y de formas de llegar al público han de ser constantes. La eficacia de las campañas publicitarias tradicionales cae en picado. "Los medios de masas tradicionales se han debilitado y los publicistas han de considerar nuevos canales y alternativas" (Iniesta-Alemán, 2019, p. 154). Originalmente, las marcas les 
ofrecían productos, pero su relación no iba más allá de probarlo y opinar públicamente si lo consideraban, no estaba profesionalizado como sí sucede actualmente. Los influencers surgieron como necesidad de hacer una comunicación más sincera y que conectara de manera más natural con el consumidor, y aunque hay algunos síntomas que indican que hay agotamiento (Ferrer-López, 2020), en este 2021 siguen siendo una fuente de referencia especialmente para los jóvenes, que prestan más atención al mensaje cuando lo prescribe un influencer y no la marca directamente en forma de anuncio, ya sea publicitario u orgánico. ${ }^{1}$

Para los jóvenes, objeto de estudio de esta publicación, Instagram es una red social de uso diario en la que interactuar con amigos y desconocidos y compartir contenido, ya sea en formato fotografía o vídeo (IAB, 2020). Muchos de esos jóvenes decidieron empezar a abrir canales en la plataforma digital de vídeos para hablar de sus temas, hacer bromas, tratar sobre videojuegos, fitness o moda. Actualmente, "muchos de ellos han convertido su hobby en un negocio que los ha hecho saltar a la fama, convirtiéndolos en ídolos de masas para millones de personas" (Rego y Romero, 2016, p. 197). Es interesante resaltar que para un $40 \%$ de los jóvenes, ser influencer se ha convertido en una aspiración, y un $64 \%$ no rechazaría la opción. Vivir nuevas experiencias, viajar, ir a restaurantes, recibir productos tecnológicos, hacer fotos..., son muchas las aficiones y experiencias que asocian con este oficio (ABC, 2019). En cambio, en aspectos más negativos de su relación con Instagram, les preocupa la imagen social que proyectan hacia el resto de usuarios, cuestión en la que implican una de las mayores cantidades de esfuerzo a la hora de construir su perfil social, la descripción del mismo y las imágenes e historias que publican en la red (Kalalo, 2018, p. 292).

Desde un punto de vista más cualitativo y que complemente los datos que ofrece Instagram, como son los me gusta, el alcance, los comentarios y las publicaciones compartidas, el neuromarketing se convierte en una herramienta para conocer más en profundidad esta relación a tres bandas entre las marcas, los jóvenes y los influencers, y en la que las emociones juegan un papel vital para lograr la eficacia comunicativa y una relación largoplacista con el usuario. "Es fundamental definir los sentimientos que queremos vender junto a los productos o servicios" (Núñez, 2021), y en esa definición tendrá peso el influencer. El neuromarketing "ha puesto el foco sobre la importancia de las emociones y los factores que influyen de manera inconsciente en la toma de decisiones por parte de los consumidores" (Canorea y Cristófol, 2018, p. 54). Si se conoce a éste, el mensaje que ofrecerá el influencer, habitualmente basado en la felicidad y en la promesa de beneficio, se adaptará mejor a la audiencia.

En esa importancia de adaptar el mensaje a las particularidades de Instagram y de cómo es la dinámica de uso, es relevante cómo se encaja la publicidad en un espacio en el que originalmente no existía y que causa cansancio a muchos usuarios (IAB, 2020). "Estimulando las emociones deseadas, por tanto útiles, transformadas en

\footnotetext{
${ }^{1}$ En publicidad, el término orgánico hace referencia a aquellas acciones comunicativas que se ejecutan usando únicamente los medios propios, es decir, que no requieren de inversión económica para amplificarlas.
} 
decisiones de compra o de voto, por lo que el conocimiento, control y manipulación del cerebro humano es un objetivo de primer orden en todos los campos" (Gómez y Patiño y Bandrés-Goldáraz, 2014, p. 408). Y dentro de esos campos, debemos incluir el de la publicidad, disciplina que basa su existencia en la persuasión y en la búsqueda del convencimiento. "La utilización de influencers podría aumentar la atención y emoción de los contenidos publicitarios, y los formatos con menos apariencia publicitaria con influencers podrían ser más efectivos que un spot tradicional protagonizado por un influencer." (Ferrer, 2020, p. 257)

Predomina en las redes sociales que el individuo cobre cierto protagonismo y pase a diluirse dentro de una red de contactos, de mayor o menor número, con la que participa y crea contenidos (De Salas, 2010). Entender sus emociones ofrecerá las respuestas adecuadas a cómo debe comportarse una marca en Instagram y qué influencers son los más adecuados para representarla. "Los partidarios del neuromarketing son firmes en la idea de que las pruebas muestran, sin temor a equívocos, que las emociones están por encima de la razón" (Pérez-Pérez, 2019:15), y las emociones son un recurso fundamental para generar atención y participación en las redes sociales. Si la acción de marketing del influencer es coherente con el contenido que publica habitualmente, sus fans reaccionan de forma más favorable y se interesan por los productos anunciados (Belanche et al., 2020). Esa congruencia tiene relación con ser fiel al estilo y al tono, aunque se trate de una colaboración pagada, y en ese estilo tienen que ver las emociones: empatía, humor, motivación, cercanía..., ofrecidos, es decir, emociones en un ecosistema digital que se nutre de sentimientos más que de contenido meramente racional.

Es imperativo concluir esta introducción haciendo referencia a la cuestión reguladora sobre la relación entre marcas e influencers. La velocidad a la que se producen los cambios en Instagram, que va desde nuevos formatos ${ }^{2}$ no proyectados en su origen (stories, reels, Instagram TV) hasta la introducción de piezas publicitarias o la ya mencionada relación contractual entre las marcas y los influencers, hace necesaria una legislación que ponga límites que protejan a todos los actores de este proceso comunicativo. Así se ha percibido en Autocontrol, que desde el 1 de enero de 2021 ha promovido la entrada en vigor de dos códigos de importancia: el Código sobre el uso de los influencers en la publicidad, elaborado de manera conjunta con la Asociación Española de Anunciantes (AEA), y el Código de tratamiento de datos en la actividad publicitaria.

\footnotetext{
2 Stories: formato para la inserción de fotos, vídeos o texto en dirección preferiblemente vertical, de veinticuatro horas de máxima duración y en la que se pueden insertar texto, música, GIF's o cajas de texto.

Reels: piezas en formato vídeo de hasta treinta segundos de duración y de exposición permanente, especialmente usados para el humor, bailes o consejos sobre un tema determinado.

Instagram TV: canal compuesto por piezas en formato vídeo de hasta una hora de duración y usado frecuentemente para compartir un contenido que necesita más tiempo para ser expuesto.
}

Vivat Academia. Revista de Comunicación. 2021, nº 154, 57-78 
En lo relativo a Instagram, el Código sobre el uso de los influencers en la publicidad (Aprobado el 9 de octubre de 2020 y firmado también por el Ministerio de Asuntos Económicos y Transformación Digital y el Ministerio de Consumo) pone especial énfasis en la obligatoriedad de identificar cuándo una mención o una publicación se tratan de acciones pactadas entre la marca y el influencer, es decir, "que responde a un acuerdo previo por el que la marca satisface una contraprestación" (Villajoana et al., 2019 , p. 116). Incluir la palabra o etiqueta identificativa en el título encima de la foto o al inicio del texto que se muestra. Si únicamente se ve una imagen, la propia imagen debe incluir la palabra o etiqueta identificativa al inicio del mensaje. También puede utilizarse la etiqueta identificativa de la publicidad establecida por la propia plataforma (Autocontrol, s.f.). Al ser una regulación muy reciente, no encontramos estudios concluyentes sobre si la aplicación de la norma se está cumpliendo. Hasta el momento, se puede afirmar que "existen indicios suficientes para considerar que la publicidad encubierta y engañosa son la norma, mientras que la transparencia en el contenido y en la naturaleza del mensaje publicitario son la excepción" (Sixto-García y Álvarez, 2020, p. 1620).

\section{OBJETIVOS}

Partiendo de este análisis previo y de su contexto, los objetivos de este estudio los podemos catalogar en dos bloques:

a) Principal: analizar el grado de interés y confianza que generan a estudiantes o graduados en Publicidad y RR.PP. los influencers cuando pactan colaboraciones con las marcas en la red social Instagram.

b) Específicos: conocer la influencia que ejercen a la hora de comprar estos preceptores de marca sobre la muestra participante. A su vez, crear un punto de partida a ampliar en estudios posteriores sobre si el hecho de estudiar o haber estudiado el Grado de Publicidad y RR.PP. influye a la hora de ser atraído por un influencer cuando muestra un producto o servicio en una publicación previamente acordada con la marca a la que representa. Escoger una muestra de publicaciones de influencers en Instagram, cuando las marcas son parte del contenido total o parcialmente, y comprobar si se especifica que se trata de una colaboración publicitaria o por el contrario no se detalla.

\section{METODOLOGÍA}

Este estudio se aborda principalmente desde los resultados que nos ofrecen las preguntas abiertas y cerradas de la encuesta ${ }^{3}$ realizada a una muestra de doscientos treinta alumnos o exalumnos de Publicidad y RR.PP. mayores de dieciocho años, menores de veintinueve y que han cursado sus estudios total o parcialmente en la Universidad de Valladolid. El motivo de la acotación por edad se debe a que Instagram es la red social mayoritaria en la que los jóvenes se relacionan con las marcas (IAB,

\footnotetext{
${ }^{3}$ Encuesta realizada a través de la plataforma Google forms.
} 
2020) y son quienes mantienen una relación más estrecha con los influencers. Para ellos son especialmente espacios de expresión, de intercambio de opiniones e ideas y de aprendizaje, en los que tienen la oportunidad de comunicarse libremente (Moreno, 2011). También sucede algo similar en otras edades, sin embargo, introducir en este estudio rangos de edades superiores puede contaminar los resultados ya que se trataría de dos tipos de público más distanciados y con intereses y conductas diferentes.

Para establecer una proporción equilibrada, de las doscientas treinta encuestas, ciento quince fueron en el rango de edad de $18-23$ años (50\%) y otras ciento quince de 24-29 años (50\%).

A su vez, como complemento a la encuesta se acompaña este estudio con un pequeño análisis de veinte publicaciones de Instagram emitidas por influencers en los meses de noviembre y diciembre de 2020 y enero de 2021, influencers que en la encuesta fueron citados. Los elementos analizados han sido: las menciones que reciben sobre el producto anunciado, las menciones negativas (de esta manera comprobamos si el producto tiene protagonismo dentro de la propia publicación) y la especificación o no de que se trata de una colaboración, hecho que puede hacer variar la percepción y el deseo generado en el espectador.

\section{RESULTADOS Y DISCUSIÓN}

En publicidad, la efectividad consiste en proporcionar verdaderas soluciones a los problemas y crear a partir de ellas nuevas realidades (Sebastián-Morillas et al., 2020). Para ello es clave el conocimiento detallado del público objetivo: en qué medios de comunicación y soportes se mueve, qué le atrae, por qué usa o puede llegar a usar nuestro producto, con qué personajes se identifica..., son algunas de las cuestiones que hay que abordar a la hora de elegir un influencer para una campaña publicitaria. Centrados ya en los jóvenes, se trata de "aprovechar la asequibilidad, accesibilidad y cercanía de la red para influir en las decisiones de compra de una nueva generación" (Pérez-Curiel, 2018, p. 258).

Son muchos los autores y autoras que previamente han estudiado la relación entre redes sociales, influencers y jóvenes, entendiendo a éstos últimos como un público amplio que puede delimitarse en función de muchas categorías para estudiarlos de forma más segmentada. Así podemos destacar los que hacen referencia a la adicción a las redes sociales, al enfoque que le dan lo jóvenes en su uso diario, la credibilidad que se le otorga, o la influencia que se ejerce con ellos a través de mensajes persuasivos (Arias-Medranda, 2017; Pérez-Curiel, 2018; Marín et al., 2015; Martín-García y ÁvilaRodríguez de Mier, 2020; Moreno, 2011; Rubio-Romero y Barón-Dulce, 2019; SánchezRodríguez et al., 2015; Sánchez-Vega et al., 2016).

Centrándonos ya en la encuesta que hemos aplicado, la primera pregunta hacía referencia a los motivos por los que los jóvenes siguen a algunas marcas en Instagram.

Vivat Academia. Revista de Comunicación. 2021, nº 154, 57-78 
El 67'8\% destaca el contenido creativo como principal motivo para seguir a una marca, reforzando un dato citado anteriormente de que los jóvenes no aceptan la publicidad tradicional en sus redes sociales. Informarse de las novedades de la marca $\left(53^{\prime} 5 \%\right)$ y por las ofertas y descuentos $(487 \%)$ completan las tres respuestas principales. Tan sólo un $10^{\prime} 4 \%$ cita entre las razones los influencers con los que trabajan las marcas que siguen. Este dato refleja que es preferible para la marca que el peso de una campaña con el influencer se reparta entre el contenido publicado en su página oficial, en este caso más como apoyo, y en la del influencer, siendo en esta segunda donde más efectiva puede ser al verse como más espontánea y menos comercial. Y más allá de los porqués, hay que conocer si la presencia de las marcas en Instagram es positiva en cuanto al grado de confianza de los encuestados. Tal como vemos en la Fig.4.1., sin ser la única razón, sí se puede afirmar que para la muestra de este estudio contar con la marca en Instagram juega a favor.

\section{¿Te genera más confianza una marca por el hecho de estar presente en Instagram? \\ 230 responses}

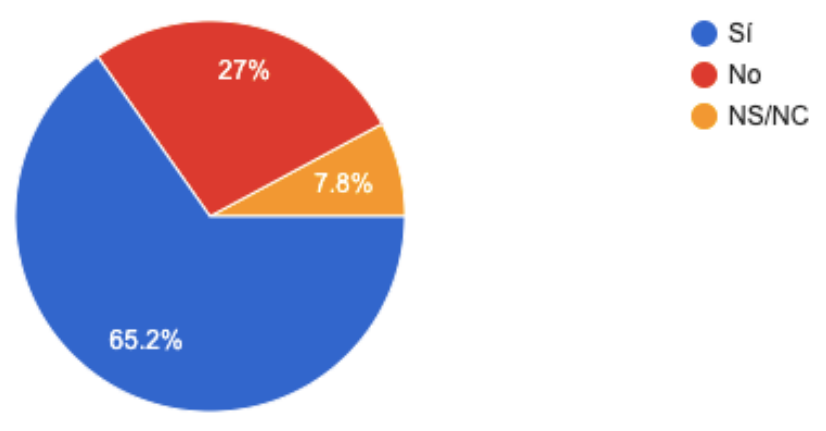

Figura 1. Grado de confianza que genera una marca en Instagram.

Fuente: elaboración propia, 2021.

Dimos la opción a continuación de que mediante una pregunta abierta nos expusieran las razones por las que confían más en una marca si está activa en Instagram. Los seis motivos principales, dentro de un abanico amplio de opciones, fueron: 1) Cercanía. 2) Estar actualizada. 3) Poder contactar con la marca. 4) La confianza que da la verificación de la cuenta por parte de Instagram (su ausencia genera incertidumbre). 5) Humaniza a la marca. 6) Los comentarios de otros usuarios como referencia.

Tratándose de una muestra formada exclusivamente por alumnos y exalumnos del Grado de Publicidad y RR.PP. o de la extinta licenciatura, no observamos una opinión mayoritaria que indique que haber cursado estos estudios lleve implícito un descenso de la confianza en el mensaje que lanza el influencer en Instagram. El plan académico actual contiene asignaturas relacionadas con el tema tratado, como Redes sociales y comunicación, Redacción publicitaria, Creatividad online, Procesos de creación del 
mensaje publicitario: medios y soportes ${ }^{4} . . .$, y conocer de forma más técnica cómo funciona una acción publicitaria entre una marca y un influencer podría tener como resultado que hubiera cierto grado de desconfianza, como así lo indica un 40'2\% de la muestra, resultado inferior a la suma de los que consideran que confían más $\left(15^{\prime} 7 \%\right)$ y de los que no le influye de ninguna manera, que son los mayoritarios con un $44^{\prime} 1 \%$.

\section{Señala la opción con la que estás más de acuerdo}

229 responses

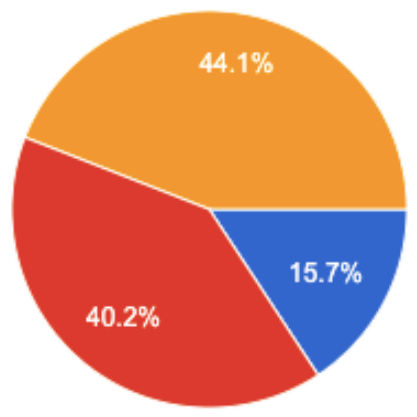

Estudiar/haber estudiado el grado en Publicidad y RR.PP. me hace confiar más en una marca cuando recurre a un influencer para emitir su mensaje

Estudiar/haber estudiado el grado en Publicidad y RR.PP. me hace confiar menos en una marca cuando recurre a un influencer para emitir su mensaje Estudiar//haber estudiado el grado en Publicidad y RR.PP. no influye en mi percepción sobre la marca cuando ap...

Figura 2. Confianza en la publicidad con influencers en relación con los estudios de Publicidad y RR.PP.

Fuente: elaboración propia, 2021.

Introduciéndonos en aspectos concretos sobre elementos más persuasivos, preguntamos por los aspectos que consideran decisivos para que el mensaje que publica un influencer sobre una marca resulte convincente en Instagram. Como vemos en los resultados de la Fig.4.3., no hay uno que sea el único decisivo, sino que un conjunto de buenas decisiones, previamente estudiadas, es lo que hace que la colaboración publicitaria sea eficaz para los actores protagonistas (marca, influencer y público). Entre estos motivos destacamos el de elegir un personaje que sea acorde a los valores de la marca a la que representa $(83,5 \%)$. Con un porcentaje alto podemos interpretar que de cara al espectador la congruencia entre el mensaje y quién lo comunica es vital para alcanza notoriedad. Que el mensaje no se perciba como excesivamente comercial (64,3\%) refuerza un hecho que hemos citado anteriormente; en una red social en la que la creatividad y el entretenimiento son factores fundamentales que motivan su crecimiento años tras año, la creación de historias que se salgan de lo más tradicional es un punto a favor de la marca. "Los formatos intermedios, los branded content, parecen que generan mayor emoción que los que son más encubiertos y con una realización más casera" (Ferrer-López, 2020, p. 257). Que el usuario se sienta identificado con el contenido que publicita $(55,2 \%)$ nos lleva a citar la importancia de una buena planificación de medios que haga que los contenidos

\footnotetext{
4 Información extraída de la web de la Universidad de Valladolid: http://bit.ly/PlanDeEstudiosPublicidad
} 
lleguen al público objetivo de la marca, es decir, al que se siente o puede llegar a sentirse interesado por el producto o servicio mencionado. Por otra parte, que el influencer no esté continuamente publicitando marcas en su página oficial $(54,8 \%)$ da indicios de que un exceso de contenido publicitario, esté reseñado como tal o no, produce saturación en los usuarios de Instagram. Por último, el quinto aspecto para que el influencer resulte convincente hace referencia a que mantenga el tono habitual de sus publicaciones no comerciales (50,9\% de los encuestados así lo cree), reafirmando la importancia de que sea la marca la que se adapte al tono del influencer, y no al revés.

¿Qué aspectos consideras decisivos para que el mensaje que publica un influencer sobre una marca resulte convincente?

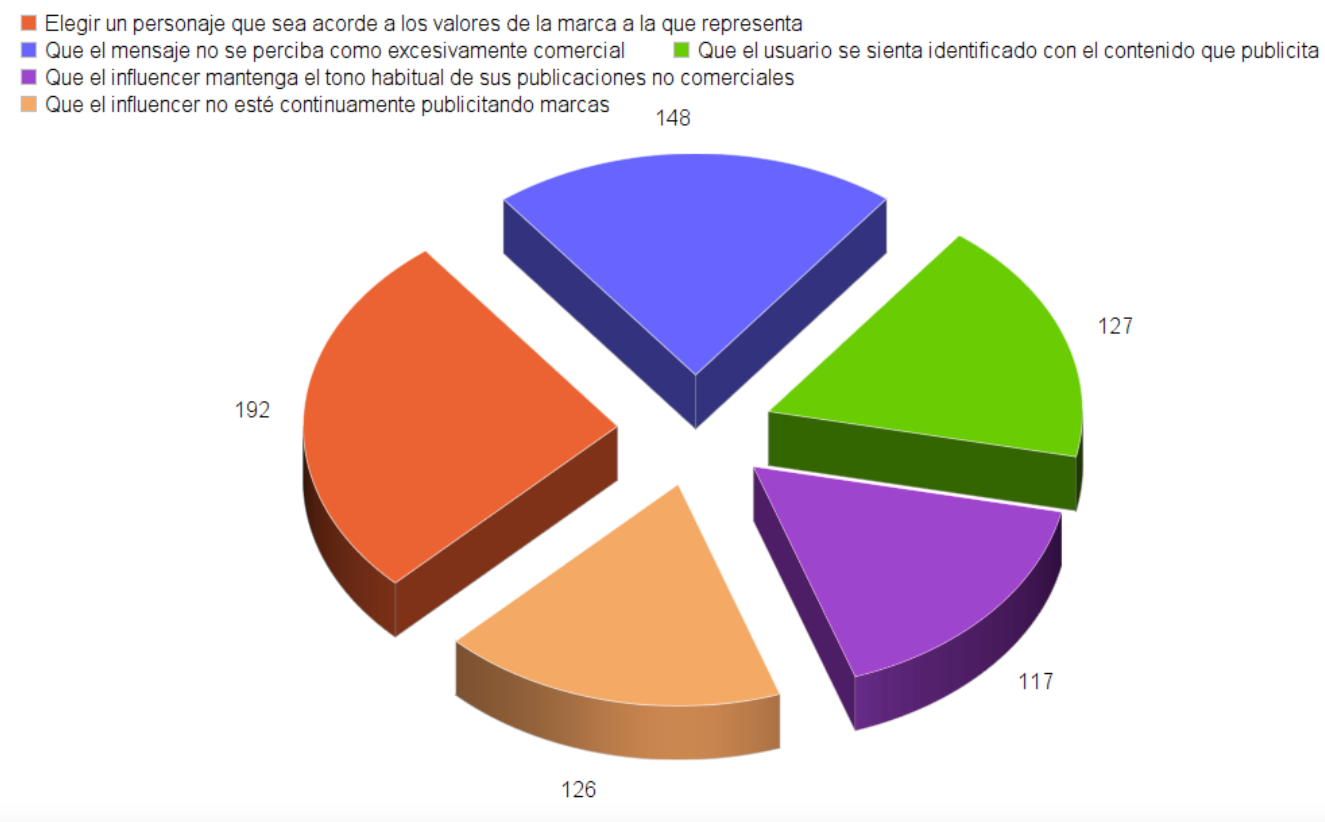

Figura 3. Aspectos por los que el mensaje del influencer es creíble.

Fuente: elaboración propia, 2021.

La pregunta anterior la complementamos con la siguiente: ¿qué aspectos consideras decisivos para que el mensaje que publica un influencer sobre una marca carezca de credibilidad en Instagram? Nos encontramos con una coherencia entre las respuestas que vemos también en la Fig.4.3. La más respondida, dentro de las opciones ofrecidas a los encuestados, es que el influencer repita demasiadas veces los beneficios del producto $(68 \%)$, correlativa a la de que el mensaje no se vuelva excesivamente comercial. Ambas, ya sean para expresar acierto o fallo respectivamente, vienen a recordarle a la marca que hay que introducir el mensaje publicitario de forma natural dentro del discurso. Un exceso de colaboraciones publicitarias del influencer $(53,9 \%)$ le perjudica tanto a él como a la marca, y esa falta de naturalidad terminará siendo notoria, con el riesgo de perder el interés de los espectadores. Que hable de la marca/producto y no especifique que se trata de una colaboración pactada, aspecto del que ya hemos hablado anteriormente en la introducción (50,9\%), no sólo acarrea problemas legales por incumplimiento de la normativa vigente, sino que se traduce en

Vivat Academia. Revista de Comunicación. 2021, n 154, 57-78 
una pérdida de credibilidad al considerarse que no se está dando un dato fundamental, el de que ha pactado previamente el contenido que está emitiendo. Un aumento de ciudadanos críticos, que se convierten no solo en espectadores sino también en emisores de contenido, ha de obligar a marcas e influencers a proyectar sus campañas siguiendo la normativa vigente y especificando, al igual que lo hacen los medios tradicionales, que se trata de publicidad.

¿Qué aspectos consideras decisivos para que el mensaje que publica un influencer sobre una marca carezca de credibilidad?

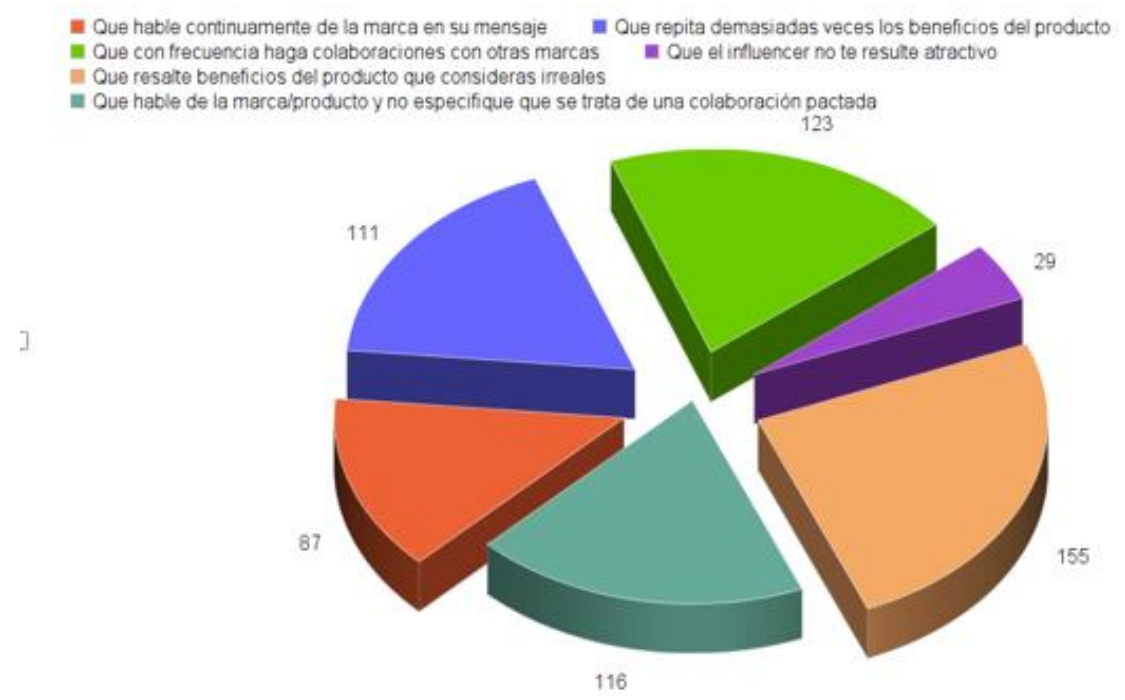

Figura 4. Aspectos por los que el mensaje del influencer pierde credibilidad.

Fuente: elaboración propia, 2021.

En la siguiente pregunta nos adentramos en el acto de compra relacionado con el mensaje difundido por el influencer, y tal como vemos a continuación en la Fig.4.5., son más los encuestados que afirman que este tipo de publicaciones que estamos analizando ha sido un condicionante que ha favorecido el consumo, dato que puede servir de punto de partida para futuros estudios más amplios que corroboren la utilidad no sólo mediática sino también comercial de los influencers. La marca que más citan los encuestados a la hora de valorar cuáles usan este recurso acertadamente es Freshly Cosmetics. 
¿Has comprado algún producto o servicio por haber sido promocionado por un influencer en Instagram?

229 responses

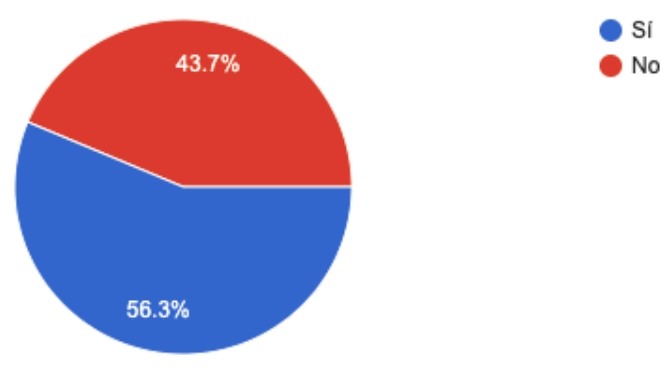

Figura 5. El influencer como condicionante del acto de comprar.

Fuente: elaboración propia, 2021.

Como reafirmación de la pregunta sobre el acto de compra, preguntamos acerca del papel que ejerce el influencer como sustituto de la marca a la hora de contar los beneficios del producto protagonista de la colaboración en Instagram. A estos prescriptores de marca se les sigue por su contenido, por su tono, por los valores que proyectan al exterior, por humor..., son múltiples factores que han hecho que los usuarios de esta red social opten por seguirle. Hay que reseñar que la llegada de las marcas a las páginas de los influencers es una consecuencia de su popularidad, no una causa. El hecho de hablar y de exponer el producto de una forma diferente y novedosa a lo que venimos estando acostumbrados en los formatos clásicos, hace que como vemos en la figura 4.6., sea mayoritaria la opción respondida de que el deseo aumenta cuando es el influencer quien habla en nombre de la marca, ya que la acerca al usuario en un formato que aún no está agotado.

Contrastamos estos datos con los del último estudio publicado por IAB (2020), en el que se afirma que un 34\% considera creíbles a los influencers que sigue (resultado parecido al 2019). Aunque la pregunta 4.6 y la que citamos no es exactamente la misma, observamos que sigue una tendencia parecida en lo referente a la credibilidad, aunque más bajos en los datos del IAB entre otros motivos porque su estudio abarca edades comprendidas entre los 16-65 años, siendo parte de esa franja un público menos afín a las redes sociales y por extensión más desconfiado y desconocedor de esta forma de comunicación digital que se da especialmente en un público más joven. 
¿Crees que cuando es el influencer el que habla en nombre de la marca aumenta tu deseo de compra del producto o servicio anunciado?

230 responses

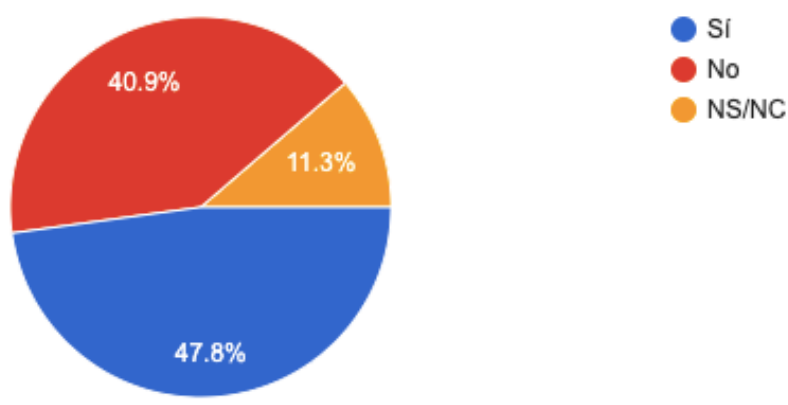

Figura 6. Relación entre el deseo de compra y la aparición del influencer.

Fuente: elaboración propia, 2021.

Volviendo a cuestiones relativas a la credibilidad, pues de la percepción que el usuario tenga acerca de ella estará en buena parte el éxito de una acción publicitaria en Instagram, preguntamos a los encuestados sobre la procedencia del mensaje. ¿Es más creíble cuando el influencer emite el contenido desde la cuenta oficial de la marca, o cuando no se sale de su hábitat y hace la publicación desde su propia página? Es frecuente ver colaboraciones en las que el influencer se queda en su propio canal, pero a su vez la marca aprovecha los contenidos y los replica en su cuenta oficial. Como observamos en la figura 4.7., la credibilidad es más eficaz si la acción se realiza preferiblemente en la cuenta del influencer $(43,2 \%)$, aunque no debemos dejar de lado, como estamos observando en varias respuestas obtenidas de esta encuesta, que sin ser mayoritarios, los datos que indican que a una parte de los internautas el recurso de los influencer no les resulta especialmente llamativo o eficaz, hay que tenerlos en cuenta y dejan margen de mejora a la creatividad, pues todavía hay un nicho que no ha sido persuadido.

Te resulta más creible un mensaje del influencer sobre la marca cuando...

222 responses

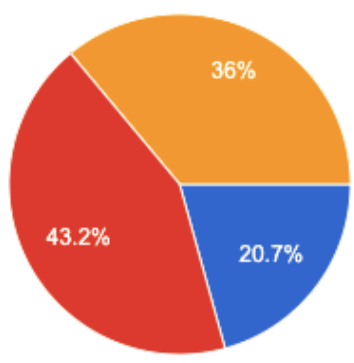

La publicación se hace en el perfil oficial de la marca

La publicación se hace en el perfil oficial del influencer

La aparición del influencer no vuelve el mensaje más creible

Figura 7. Credibilidad del mensaje en función de su procedencia.

Vivat Academia. Revista de Comunicación. 2021, nº 154, 57-78 
Fuente: elaboración propia, 2021.

Finalizamos esta encuesta con la figura .4.8. Observamos una preocupante percepción de que la publicidad con influencers está saturada debido al exceso de impactos que reciben día a día en el tiempo que pasan conectados a Instagram. Esta saturación puede conllevar la pérdida de seguidores de las marcas en sus páginas oficiales de Instagram en caso de que perciban que la calidad y las temáticas de los contenidos no van en relación a sus intereses, citados en este artículo, tales como la creatividad y los contenidos de calidad.
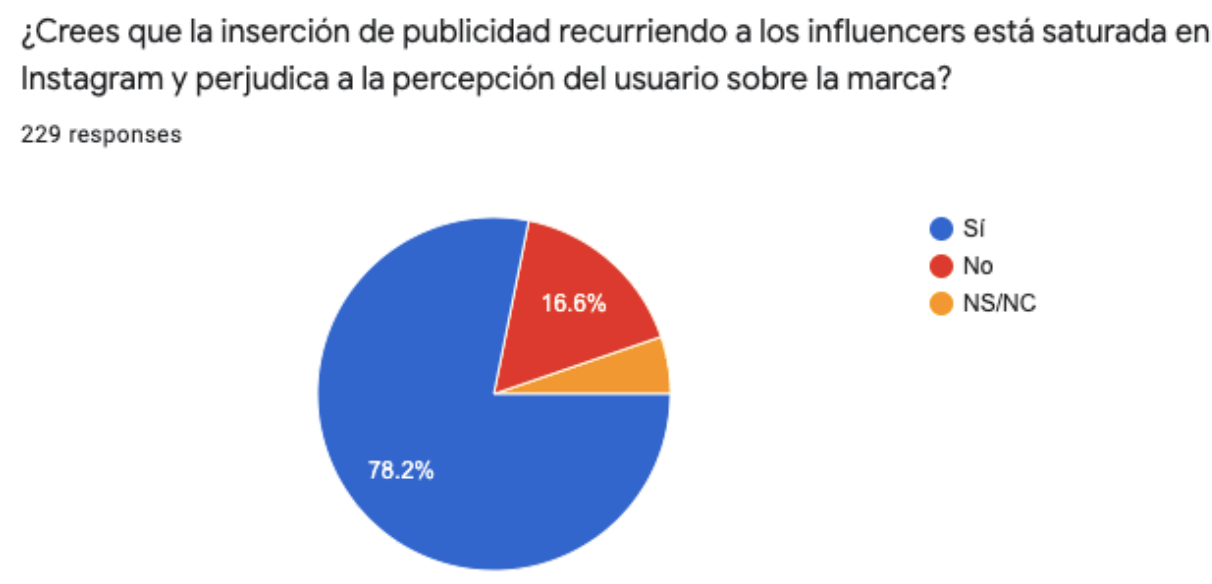

Figura 8. Percepción sobre la saturación de acciones de marca con influencers.

Fuente: elaboración propia, 2021.

\section{Una breve aproximación a la dinámica real de los influencers en Instagram.}

Para acompañar a este estudio y tener una visión más completa del panorama mediático que estamos tratando, hemos elegido cinco influencers ${ }^{5}$ de los mencionados por los participantes de la encuesta y que consideran que hacen una buena comunicación. Estos son: María Pombo, Noemí Salazar, Jonan Wiergo, Laura Escanes y Rocío Osorno.

\footnotetext{
5 Cuentas oficiales de los influencers en Instagram: @lauraescanes, @jonanwiergo, @mariapombo, @noemi_salazar13, @rocio0sorno,
} 


\begin{tabular}{|c|c|c|c|c|c|c|}
\hline INFLUENCER & №fans & Marca/Producto & Objetivo delacolaboración & Especificalacolaboración & Mencionesdelosfanssobrelamarca & Mensajesnegativossobrelamarca \\
\hline \begin{tabular}{|l|} 
Laura Escanes \\
\end{tabular} & 1'5 millones & & & & & \\
\hline Post 1 & & Amazon & Branding y participación & si & $1 /+100$ & 1 \\
\hline Post 2 & & Multiópticas & Sorteo + Tráfico & si & $0 /+100$ & NO \\
\hline Post 3 & & Adidas & Beneficios producto (Vídeo) & si & $46 /+100$ & 3 \\
\hline Post 4 & & Women Secret & Beneficios producto (Vídeo) & si & $25 /+100$ & 3 \\
\hline \begin{tabular}{|l|} 
Noemi Salazar \\
\end{tabular} & 865.000 & & & & & \\
\hline Post 1 & & Sit (mudanzas) & Beneficios producto & si & $0 /+100$ & NO \\
\hline Post 2 & & Shein (moda) & Descuentos/Tráfico web & NO & $2 /+100$ & NO \\
\hline Post 3 & & Glia (cosméticos) & Descuentos/Tráfico web & NO & $1 /+100$ & NO \\
\hline Post 4 & & Cortinadecor (cortinas) & Tráfico web/Beneficios marca & NO & $3 /+100$ & NO \\
\hline \begin{tabular}{|l|} 
Jonan Wiergo \\
\end{tabular} & 642.000 & & & & & \\
\hline Post 1 & & Sting (gafas de sol) & Sorteo + nuevos fans & NO & $0 /+100$ & No \\
\hline Post 2 & & Jean Paul Gaultier & Beneficios producto & NO & $4 /+100$ & 1 \\
\hline Post 3 & & Be Mate (alquiler apartamentos) & Beneficios producto & NO & $0 /+100$ & NO \\
\hline Post 4 & & Calvin Klein (ropa interior) & Beneficios producto & si & $2 /+100$ & NO \\
\hline \begin{tabular}{|l|} 
Rocío Osorno \\
\end{tabular} & 1'3 millones & & & & & \\
\hline Post 1 & & Gisèle Denis (perfume) & Sorteo + nuevos fans & NO & $0 /+100$ & NO \\
\hline Post 2 & & (depilación)Centro Laserum & Sorteo + nuevos fans + oferta & NO & $0 /+100$ & NO \\
\hline Post 3 & & Oysho (ropa) & Beneficios producto & NO & $3 /+100$ & NO \\
\hline Post 4 & & Centro María Hernández (estética) & Beneficios producto (vídeo) & NO & $2 /+100$ & NO \\
\hline María Pombo & 1'8 millones & & & & & \\
\hline Post 1 & & Intimissimi (ropa interior) & Beneficios producto & si & $9 /+100$ & NO \\
\hline Post 2 & & Maskk (mascarillas y moda) & Beneficios producto & si & $9 /+100$ & 3 \\
\hline Post 3 & & Mango & Beneficios producto & si & $14 /+100$ & 3 \\
\hline Post 4 & & Mango & Beneficios producto & si & $18 /+100$ & NO \\
\hline
\end{tabular}

Figura 9. Tabla de contenido de los influencers seleccionados en Instagram.

Fuente: elaboración propia, 2021.

Las condiciones que tenían que cumplir para acceder a esta muestra a analizar han sido: 1) Haber sido mencionados por los participantes en la encuesta. 2) Tener una comunidad de fans de al menos seiscientos mil seguidores. 3) Hacer colaboraciones regularmente con las marcas. 4) Haber publicado al menos cinco colaboraciones en los últimos tres meses (noviembre y diciembre de 2020, y enero de 2021). 5) Que en todas las publicaciones publicitarias tengan al menos cien respuestas.

Para conocer el grado de confianza de los seguidores de los influencers con respecto a las colaboraciones que hacen con las marcas, no es adecuado sacar conclusiones recurriendo a los comentarios que los fans dejan en cada publicación, pues como observamos en la figura 9., apenas hablan sobre el producto mostrado; su presencia es testimonial ya que todo el protagonismo se lo lleva el influencer y las respuestas que recibe son relativas a su persona, apenas hay interés directo en este formato de interacción. Sin embargo, tampoco concluimos que estas acciones no generen beneficios para las marcas porque el objetivo de gran parte de las publicaciones vemos que es mostrar el producto, darle notoriedad mostrando sus beneficios y que se asocien a los valores que desprende el prescriptor, no a un proceso de venta cortoplacista.

No encontramos una posición común a la hora de especificar en las publicaciones que se tratan de colaboraciones pagadas. Únicamente Laura Escanes recurre al formato que ofrece Instagram de detallar la colaboración con la etiqueta predestinada a ello debajo del nombre de usuario. En el caso de María Pombo, sí que lo menciona, pero únicamente poniendo en el texto el hashtag \#AD, que queda en segundo plano. Recordamos que los encuestados valoran negativamente que los influencers encubran sus contenidos publicitarios sin contarle a la audiencia que es una colaboración. En cuanto a Wiergo,

Vivat Academia. Revista de Comunicación. 2021, n 154, 57-78 
Salazar y Osorno, sus publicaciones no dejan claro con los recursos disponibles que trabajan con las marcas.

Las redes sociales son un medio de comunicación bidireccional en las que los consumidores de una marca pueden expresar libremente sus opiniones. Esto ha motivado que sea frecuente encontrar en las páginas oficiales críticas negativas sobre la calidad, la gestión, el precio, el servicio de atención al cliente... Pero como vemos en este apartado, cuando son los influencers los que citan a las marcas, apenas vemos comentarios negativos contra ellas. Estar bajo el abrigo de personas que gozan de carisma y son queridas en las redes sociales hacen de estas acciones comunicativas espacios alejados del conflicto.

\section{CONCLUSIONES}

Los encuestados siguen a las marcas en Instagram con el objetivo de obtener un beneficio, que tienen que adaptarse al uso y al lenguaje predominante en Instagram y ofrecer contenido de calidad que vaya más allá de lo estrictamente comercial.

La transparencia comienza por el mero hecho de estar presente en las redes sociales, de exponerse en este caso en Instagram, y convertirla en una herramienta bidireccional en la que tanto marca como usuario puedan aportar contenido y formen parte de una conversación de la que ambos salen beneficiados. Escuchar a los seguidores y dar salida a sus dudas crea cercanía y la humaniza.

No observamos una tendencia excesivamente mayoritaria sobre si el hecho de estudiar o haber estudiado Publicidad y RR.PP. condiciona la percepción que tienen los encuestados sobre los mensajes que publican los influencers hablando de marcas. Dejamos abierta la posibilidad de abordar más en profundidad este campo en futuros estudios y establecer relaciones con el contenido sobre el tema que se imparte en el plan de estudios de la titulación e incluso compararla con otros estudios universitarios para concretar más si esa percepción es real o no.

La elección de un influencer no debe responder únicamente a criterios cuantitativos. Que el personaje sea acorde a los valores que proyecta la marca, que no se exponga continuamente a la publicidad o que el mensaje vaya más orientado a consolidar la identificación del consumidor potencial, es lo que generará credibilidad, el verdadero objetivo de la publicidad, y que aumentará las opciones de una acción comercial posterior. Por el contrario, si en el contenido del influencer elegido se pone demasiado énfasis en los beneficios del producto o éstos los exagera, se producirá un descenso en dicha credibilidad. Encajar el producto dentro del tono de comunicación que suele usar el influencer es clave. A su vez, por cuestiones legales y por transparencia con sus fans, todas las compañías deberían reseñar cuándo la mención responde a un acuerdo de colaboración previamente pactado. 
De la encuesta concluimos que el poder de un influencer para motivar el acto de compra de un seguidor suyo es medianamente alto si se recurre a una buena estrategia comunicativa y de contenidos más allá del formato publicitario clásico. El papel de los contenidos es principalmente generar interés por parte de los usuarios y seguidores, y construir confianza, estrechando lazos, para fidelizar a la audiencia, con lo que finalmente se generan oportunidades de negocio sin que previamente se haya lanzado un mensaje de carácter comercial (Harad, 2013, p. 18). Ceder al influencer el peso de la mención de la marca en Instagram, que está enfocada a contenidos creativos, es un acierto, pero se ha de tener en cuenta que este tipo de acciones comunicativas tienen que ser parte de una campaña de 360 grados en la que se recurra a otros formatos publicitarios que refuercen el mensaje. De la muestra de cinco influencers que hemos analizado, observamos que, en el apartado de comentarios, las menciones recibidas sobre el producto publicitado, son testimoniales y todo el protagonismo se lo queda el propio influencer, motivo real por el que se le sigue en Instagram.

En el apartado de este estudio donde vemos una posición mayoritaria es en el de la creencia de que la publicidad en Instagram recurriendo a influencers está saturada. Al contenido que de forma orgánica publican estos prescriptores, se le suma los formatos publicitarios de pago, que en ocasiones ocupan hasta un $23 \%$ de todas las publicaciones que visualiza un usuario (Puro Marketing, 2019), y este exceso de información comercial produce rechazo en los usuarios de esta red social y obliga a la marca a buscar nuevas formas de comunicar más creativas que la hagan destacar de la competencia.

Para los estudiantes y exestudiantes del Grado de Publicidad y RR.PP., los influencer son un recurso aceptado dentro de la dinámica de consumo de Instagram, pero de la encuesta deducimos que esa aceptación no lleva implícito un cheque en blanco, y que las colaboraciones con los anunciantes deben ser creativas, espaciadas en el tiempo, coherentes con el tono de comunicación del influencer y han de estar correctamente especificadas para evitar confusiones o la lógica sensación de engaño.

\section{REFERENCIAS}

ABC. (14 de abril de 2019). Casi el 40\% de los jóvenes españoles se siente atraído por la idea de ser influencer. http://bit.ly/ABCInfluencersYJovenes

Arias-Medranda, J. M. (2017). Enfoque de las redes sociales en universitarios. Dominio de las ciencias,

(3),

186-199.

http://dx.doi.org/10.23857/dom.cien.pocaip.2017.3.mono1.ago.186-199

Autocontrol. (s.f.). Entran en vigor el Código de influencers y el Código de protección de datos. http://bit.ly/SobreAutocontrol

Vivat Academia. Revista de Comunicación. 2021, nº 154, 57-78 
Belanche, D., Flavián, M., e Ibáñez-Sánchez, S. (2020). Followers' reactions to influencers' Instagram posts, Spanish Journal of Marketing - ESIC, 1(24), 3754. https:// doi.org/10.1108/SJME-11-2019-0100

Business Instagram (s.f.). Consolida tu empresa en Instagram. http://bit.ly/FormatosPubIG

Canorea-Tiralaso, H., y Cristófol-Rodríguez, C. (2018) La felicidad en publicidad desde la perspectiva del neuromarketing. Revista de la Asociación Española de Investigación de la Comunicación, 5(10), 46-56. https://doi.org/10.24137/raeic.5.10.21

Casado, C., y Carbonell, X. (2018). La influencia de la personalidad en el uso de Instagram. Aloma, 36(2), 23-31. https://doi.org/10.51698/aloma.2018.36.2.23-31

Castelló-Martínez, A., y Del Pino Romero, C. (2019). La comunicación publicitaria con Influencers. Redmarka. Revista de Marketing Aplicado, 1(014), 21-50. http://hdl.handle.net/2183/22922

De Salas-Nestares, M. I. (2010). La publicidad en las redes sociales: de lo invasivo a lo consentido. Icono14, 8(1), 5 .

Fernández-Lerma, A. (2017). Estudio del origen de la figura del influencer y análisis de su poder de influencia en base a sus comunidades. Universidad Pompeu Fabra. http://hdl.handle.net/10230/36313

Ferrer-López, M. (2020). Neuromarketing y la medición del efecto de la publicidad de influencers en adolescentes. Revista Mediterránea de Comunicación. 2020, 11(2), 241-259. https://doi.org/10.14198/MEDCOM2020.11.2.11

García, L. (2018). ¿Qué es un influencer? 40defiebre. https://doi.org/10.5209/esmp.66921

García, Y. (2020). Tipos de influencers según su tamaño: cuatro opciones para potenciar tus campañas en redes sociales. Marketing 4commerce. http:/ / bit.ly/TiposDeInfluencers

Gómez y Patiño, M. \& Bandrés Goldáraz, E. (2014). El neuromarketing: una nueva disciplina para la investigación de audiencias y de la opinión pública, Icono 14, (12), 395-415. https://doi.org/10.7195/ri14.v12i2.666

Harad, K. C. (2013). Content Marketing Strategies to Educate and Entertain. Journal of Financial Planning, 26, 18-20. 10.4108/eai.18-7-2019.2287839 
Hernández-Fernández, A. (2013). Estrategia de comunicación de marca en las redes sociales. TIC: cuadernos de desarrollo aplicados a las TIC, 2(3). https://doi.org/10.17993/3ctic.2013.23.\%20

Iniesta-Alemán, I. (2019). Eficacia de los influencers como recurso publicitario en la estrategia de los anunciantes locales. Ámbitos. Revista Internacional de Comunicación, 46, 149-169. https://doi.org/10.12795/Ambitos.2019.i46.09

International Advertising Bureau (2020). Estudio de redes sociales 2020. IAB Spain.

Kalalo, C. N. (2018). Online self-presentation relationship with Instagram addiction in students of the department of physical education, health and recreation, university of musamus, Merauke, Indonesia. International Journal of Mechanical Engineering and Technology, 9(10), 288-294.

Kotler, P. \& Armstrong, G. (2014). Principles of Marketing. (16 ed.). Pearson.

Marín, V., Sampedro, B. E., y Muñoz, J. M. (2015). ¿Son adictos a las redes sociales los estudiantes universitarios? Revista Complutense de Educación, 26, 233-251. http://dx.doi.org/10.5209/rev_RCED.2015.v26.46659

Martín-García, N., y Ávila-Rodríguez de Mier, B. (2020). La credibilidad publicitaria en la nueva esfera mediática: los universitarios y los medios. Ámbitos: Revista internacional de comunicación, 50, 208-223. https://doi.org/10.12795/Ambitos.2020.i50.14

Morales, P. (2019). Los influencers en el protocolo. Un caso de estudio. Revista $\begin{array}{llll}\text { Estudios } \quad \text { Institucionales, 67-78. } & \text { 6(4), }\end{array}$ https://doi.org/10.5944/eeii.vol.4.n.6.2017.18826

Moreno, M. (2011). El sentido de vida en los jóvenes: redes sociales, relaciones significativas y actividades de ocio. Revista de estudios de juventud.

Navío-Navarro, M. (2021). Contenidos eficientes en redes sociales: la promoción de series de Netflix. index.comunicación, 11(1), 239-270. https://doi.org/10.33732/ixc/11/01Conten

Núñez, J. (2021). Neuromarketing o cómo utilizar la ciencia para triunfar en el comercio electrónico. El País. http://bit.ly/NeuroMKYComercioElectronico

Pérez-Curiel, C. (2018). El marketing de influencia en moda. Estudio del nuevo modelo de consumo en Instagram de los millennials universitarios. adComunica: revista científica de estrategias, tendencias e innovación en comunicación, 15, 255-281. http://dx.doi.org/10.6035/2174-0992.2018.15.13 
Pérez-Pérez, R. M. (2019). La investigación científica como motor para el éxito de la publicidad. Ciencia y 11-23. https://doi.org/10.22206/CYS.2019.V44I2.PP11-23

Puro Marketing (31 de julio de 2019). ¿Tiene Instagram ya demasiada publicidad? http://bit.ly/SaturacionEnInstagram

Rego, S. y Romero, L. M. (2016). Representación discursiva y lenguaje de los youtubers españoles: estudio de caso de los gamers más populares. Index Comunicación, 1(6), 197-224.

http://journals.sfu.ca/indexcomunicacion/index.php/indexcomunicacion/article $/$ view $/ 271 / 231$

Romero, J. M., Campos, N. y Gómez, G. (2019). Follow me y dame like. Hábitos de uso de Instagram de los futuros maestros. Revista interuniversitaria de formación del profesorado, 94, 83-96.

Rubio-Romero, J., y Barón-Dulce, G. (2019). Actitudes de los jóvenes hacia las comunidades virtuales y su vínculo con las marcas. Una aproximación a través de los estudiantes universitarios de comunicación y de marketing de la Universidad Nebrija. AdComunica, 2019, 18, 41-62. https://doi.org/10.6035/21740992.2019.18.4

Sánchez-Rodríguez, J., Ruiz-Palmero, J. y Sánchez Rivas, E. (2015). Uso problemático de las redes sociales en estudiantes universitarios. Revista Complutense De Educación, 26, 159-174. https://doi.org/10.5209/rev_RCED.2015.v26.46360

Sánchez-Vega, E., Cantero-Téllez, R. y Rodríguez-Martínez, M. del C. (2016). Adicción a Internet en futuros profesores. Un estudio de caso con alumnado universitario. Innoeduca. International Journal of Technology and Educational Innovation, 2(1), 46-53. https://doi.org/10.20548/innoeduca.2016.v2i1.1063

Sebastián-Morillas, A., Martín-Soladana, I., y Clemente-Mediavilla, J. (2020). Importancia de los 'insights' en el proceso estratégico y creativo de las campañas publicitarias. Estudios Sobre El Mensaje Periodístico, 26(1), 339-348 https://doi.org/10.5209/esmp.66570

Sheldon, P. \& Bryant, K. (2016). Instagram: Motives for its use and relationship to narcissism and contextual age. Computers in Human Behavior, 58, 89-97. https://doi.org/10.1016/j.chb.2015.12.059

Sixto-García, J., y Álvarez-Vázquez, A. (2020). Influencers en Instagram y publicidad engañosa: la necesidad de regular y autorregular. Estudios Sobre El Mensaje Periodístico, 26(4), 1611-1622. https:// doi.org/10.5209/esmp.66921 
Universidad Oberta de Catalunya. (21 de febrero de 2018). Cómo definir una estrategia de comunicación con un influencer. http://bit.ly/EstrategiaConInfluencers

Vilajoana, S., Rom-Rodríguez, J., y Miotto, G. (2019). Retos de la autorregulación publicitaria ante los riesgos jurídicos y éticos del marketing de influencers. Revista $\begin{array}{lllll}\text { Mediterránea de } & 10 & \text { (2), } & \text { 115-129. }\end{array}$ https://doi.org/10.14198/MEDCOM2019.10.2.15

\section{AUTOR:}

\section{Alberto Martín García}

Licenciado en Publicidad y RR.PP. por la Universidad de Valladolid. Compagina desde hace diez años su trabajo de community manager freelance con el de profesor asociado en el Campus María Zambrano de Segovia, donde imparte las asignaturas Estructura del Sistema Publicitario y Comunicación, sociedad y educación, en el Grado de Publicidad y RR.PP. Se encuentra realizando la tesis doctoral sobre el mundo de las redes sociales en el ámbito de la educación mediática.

alberto.martin.garcia@uva.es

ORCID: https:// orcid.org/0000-0003-2254-8811 